\title{
Simulation of transitional flows through a turbine blade cascade with heat transfer for various flow conditions
}

\author{
Petr Straka ${ }^{1, a}$, Jaromír Příhoda ${ }^{2}$, Martin Kožíšek $^{3}$, Jiř́ Fürst ${ }^{3}$ \\ ${ }^{1}$ Aerospace Research and Test Establishment, Plc, Prague, Czech Republic \\ ${ }^{2}$ Institute of Thermomechanics AS CR, v.v.i., Prague, Czech Republic \\ ${ }^{3}$ Faculty of Mechanical Engineering, Czech Technical University in Prague, Czech Republic
}

\begin{abstract}
The contribution deals with the simulation of the transitional flows with heat transfer by means the EARSM turbulence model of Hellsten [1] completed by the algebraic transition model of Straka and Př́hoda [2] and by the three-equation model of Walters and Cokjlat [3]. The both mathematical models were tested for the flat plate flow on a heated wall measured by Sohn and Reshotko [16] and then applied to the simulation of compressible flow through the VKI turbine blade cascade according to measurements of Arts et al. [4]. The simulations were carried out for subsonic and transonic regimes at various free-stream turbulence levels. The best agreement of numerical results with experimental data was achieved by the URANS approach applied for the EARSM model with the algebraic transition model giving good results for both subsonic and transonic regimes as well.
\end{abstract}

\section{Introduction}

The numerical simulation of compressible flows in turbomachinery by means of the RANS approach should be based not only on the advanced turbulence model but also on adequate models of the laminar/turbulent transition and the turbulent heat transfer. The bypass transition models come out mostly from the intermittency concept using the algebraic and/or transport equation for the intermittency coefficient, see e.g. Thermann and Niehuis [5], Straka and Př́hoda [2] and/or Langtry and Menter [6], Kubacki et al. [7], and Menter et al. [8]. Besides, the three-equation $k-k L-\omega$ model by Walters and Cokljat [3] with the equation for the energy of non-turbulent fluctuations can be used for transition modelling as well. Nevertheless both types of transition models need empirical relations describing the onset and the length of the transition region. The turbulent heat transfer is mainly modelled by the simplest assumption of the constant turbulent Prandtl number.

The contribution deals with the numerical simulation of the compressible flow through a turbine blade cascade with heat transfer by means two different turbulence models. The EARSM turbulence model of Hellsten [1] was completed by the algebraic bypass transition model of Straka and Př́ihoda [2] and by the model of turbulent heat transfer based on the generalized gradient hypothesis of Launder [9]. Futher the $k-k L-\omega$ model of Walters and Cokjlat [3] where the relation for the turbulent thermal diffusivity corresponds to the variable turbulent Prandtl number was used. Both models were tested by means of the flow over a heated flat plate and applied for simulation of flow through a turbine blade cascade.

\section{Mathematical model}

The governing equations for compressible flow are given by the Favre-averaged Navier-Stokes equations and by constitutive relations. The system of equations was closed partly by the EARSM model with the algebraic transition model implemented into the in-house numerical code and partly by the three-equation $k-k L-\omega$ model implemented into the open-source code OpenFOAM.

The EARSM model is used in the form corresponding to turbulent-viscosity models with the turbulent stress given by the relation

$$
\tau_{i j}=\mu_{t}\left(2 S_{i j}-\frac{2}{3} \delta_{i j} \frac{\partial U_{k}}{\partial x_{k}}\right)-\frac{2}{3} \delta_{i j} \rho k-a_{i j}^{(e x)} \rho k
$$

where the extra-anisotropy tensor $a_{i j}^{(e x)}$ is dependent on strain-rate and vorticity tensors and their invariants. Transport equations for the turbulent energy $k$ and the specific dissipation rate $\omega$ are given by the SST model according to Menter [11] in the form

$$
\begin{gathered}
\frac{\partial(\rho k)}{\partial t}+\frac{\partial\left(\rho U_{j} k\right)}{\partial x_{j}}=P_{k}+\frac{\partial}{\partial x_{j}}\left[\left(\mu+\sigma_{k} \mu_{t}\right) \frac{\partial k}{\partial x_{j}}\right] \\
-\beta^{*} \rho \omega k \\
\frac{\partial(\rho \omega)}{\partial t}+\frac{\partial\left(\rho U_{j} \omega\right)}{\partial x_{j}}=\frac{\omega}{k} P_{k}-\beta \rho \omega^{2}+ \\
\frac{\partial}{\partial x_{j}}\left[\left(\mu+\sigma_{\omega} \mu_{t}\right) \frac{\partial \omega}{\partial x_{j}}\right]+C_{D} \\
\text { Turbulent viscosity is given by the relation } \\
\mu_{t}=C_{\mu} \rho k \tau_{t}
\end{gathered}
$$

with the turbulent time scale

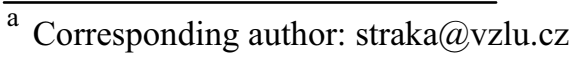




$$
\tau_{t}=\max \left(\frac{1}{\beta^{*} \omega} ; C_{\tau} \sqrt{\frac{v}{\beta^{*} \omega k}}\right)
$$

where the Kolmogorov viscous time scale is used near the wall and $\beta^{*}=0.09$ and $C_{\tau}=6$. The coefficient $C_{\mu}$ is obtained from the equation

$$
C_{\mu}=-\left(\beta_{1}+I I_{\Omega} \beta_{6}\right) / 2
$$

where coefficients $\beta_{1}$ and $\beta_{6}$ depend on strain-rate and vorticity tensors and their invariants $I I_{S}$ and $I I_{\Omega}$. The detailed description of the EARSM model is given by Hellsten [1].

The production term in the turbulent energy equation was modified for the reduction of the undesirable overproduction of the turbulent energy in the stagnation region.

The turbulent heat transfer is modelled according to Launder [9] by the generalized gradient hypothesis in the form

$$
q_{i}=-C_{t} \frac{\tau_{i j}}{\omega} \frac{\partial T}{\partial x_{j}}
$$

with the constant $C_{t}=0.3$. For the prediction of the transitional flows, the production and destruction terms in the $k$-equation are multiplied by the intermittency coefficient $\gamma$. Similarly, the effective viscosity is given by $\mu_{e f}=\mu+\gamma \mu_{t}$ in the transition region. The transition model is based on the concept of different values of the intermittency coefficient in the boundary layer $\gamma_{i}$ and in the free stream $\gamma_{e}$. The intermittency coefficient in the boundary layer $\gamma_{i}$ is expressed by the relation

$$
\gamma_{i}=1-\exp \left[-\hat{n} \sigma\left(R e_{x}-R e_{x t}\right)^{2}\right]
$$

The transition onset is given by the empirical correlation for the momentum Reynolds number $R e_{\theta t}=\mathrm{f}\left(T u, \lambda_{t}\right)$ where $T u(\%)$ is the free-stream turbulence level and $\lambda_{t}$ is the pressure-gradient parameter. The length of the transition region is expressed using the parameter $N=\hat{n} \sigma \operatorname{Re}_{\theta t}{ }^{3}$ where $\hat{n}$ is the spot generation rate and $\sigma$ is the spot propagation rate introduced by Narasimha [12]. The effect of the free-stream turbulence and the pressure gradient on the parameter $N$ is correlated by an empirical relation proposed for the attached flow by Solomon et al. [13]. The onset of transition in separated flow is given by the correlation proposed by Mayle [14] in the form $R e_{x t}=\mathrm{f}\left(R e_{\theta s}, R e_{x s}\right) \quad$ where $R e_{\theta s}$ is the momentum Reynolds number at the separation and $R e_{x s}$ is the Reynolds number related to the distance of the separation from the leading edge.

The application of local variables is necessary for prediction of transitional flows in complex geometries. Therefore the momentum Reynolds number is replaced by the maximum of the vorticity Reynolds number according to Langtry and Menter [6]. The vorticity Reynolds number is given by the relation

$$
R e_{\Omega}=y^{2}|\Omega| / v
$$

where $y$ is the distance from the wall and $\Omega$ is the absolute value of the vorticity tensor. The link between both Reynolds numbers is expressed by the relation $R e_{\theta}=$ $R e_{\Omega \max } / C$ where the parameter $C$ depends on the pressure gradient expressed by the parameter

$$
L=R e_{\Omega}^{2} \frac{v}{U_{e}^{2}} \frac{\mathrm{d} U_{e}}{\mathrm{~d} x}
$$

The three-equation model of Walters and Cokljat [2] is based on the assumption that the transition process can be expressed by the transfer from the energy of nonturbulent velocity fluctuations $k_{L}$ to the turbulent energy $k_{T}$ of three-dimensional turbulent velocity fluctuations.

The transport equations for the turbulent energy $k_{T}$, laminar energy $k_{L}$ and the specific dissipation rate $\omega$ are given by equations

$$
\begin{gathered}
\frac{D\left(\rho k_{T}\right)}{D t}=\rho\left(P_{k T}+R_{B P}+R_{N A T}-\omega k_{T}-D_{T}\right) \\
+\frac{\partial}{\partial x_{j}}\left[\left(\mu+\frac{\rho \alpha_{T}}{\sigma_{k}}\right) \frac{\partial k_{T}}{\partial x_{j}}\right] \\
\frac{D\left(\rho k_{L}\right)}{D t}=\rho\left(P_{k L}-R_{B P}-R_{N A T}-D_{L}\right)+\frac{\partial}{\partial x_{j}}\left(\mu \frac{\partial k_{L}}{\partial x_{j}}\right) \\
\frac{D(\rho \omega)}{D t}=C_{\omega 1} \frac{\rho \omega}{k_{T}} P_{k T}+\left(\frac{C_{\omega R}}{f_{w}}-1\right) \frac{\rho \omega}{k_{T}}\left(R_{B P}+R_{N A T}\right) \\
-C_{\omega 2} \rho \omega^{2}+C_{\omega 3} f_{\omega} \alpha_{T} f_{w}^{2} \rho \frac{\sqrt{k_{T}}}{y^{3}}+\frac{\partial}{\partial x_{j}}\left[\left(\mu+\frac{\rho \alpha_{T}}{\sigma_{\omega}}\right) \frac{\partial \omega}{\partial x_{j}}\right]
\end{gathered}
$$

The terms $P_{k T}$ and $P_{k L}$ express the production of turbulent and non-turbulent velocity fluctuations respectively. Similarly, terms $D_{T}$ and $D_{L}$ express the diffusion. The transfer of energy between non-turbulent and turbulent vortices is modelled by terms $R_{B P}$ and $R_{N A T}$ expressing the effect of the decay of laminar fluctuations during natural and bypass transitions. The turbulent heat transfer in the energy equation is expressed in the form

$$
q_{i}=-\rho \alpha_{\theta} \frac{\partial h}{\partial x_{i}}
$$

where $h$ is the enthalpy and $\alpha_{\theta}$ is the turbulent thermal diffusivity used in the form corresponding to the variable turbulent Prandtl number. The both mathematical models are described in detail by Fürst et al. [15].

\section{Results}

The mathematical models were tested using the transitional boundary layer on a heated flat plate according to Sohn and Reshotko [16]. Measurements were carried out in a low-speed closed-circuit wind tunnel. The heated flat plate was formed by the lower wall of the test section with the suction of boundary layer upstream of the leading edge. Measurements were accomplished at the mean velocity $U_{e} \approx 31 \mathrm{~m} / \mathrm{s}$ and the constant wall heat flux $q_{w} \approx 410 \mathrm{~W} / \mathrm{m}^{2}$ for several free-stream turbulence levels increased by grids. The initial unheated length of the flat plate was $x_{o}=0.035 \mathrm{~m}$.

For simulations were used experimental data for the grid 2 with nominal free-stream turbulence $T u=2.4 \%$. The computational domain corresponds to the experimental arrangement. The rectangular computational domain was $1.5 \mathrm{~m}$ long and $0.15 \mathrm{~m}$ high. The inlet was in the distance $x=-0.05 \mathrm{~m}$ upstream of the leading edge. Standard inlet and outlet boundary conditions were applied. The symmetry boundary condition was used on 
the upper boundary of the domain. The constant temperature gradient was prescribed at the wall according to the Fourier law $q_{w}=\lambda(\partial T / \partial y)_{\mathrm{w}}$ where $\lambda$ is the thermal conductivity.

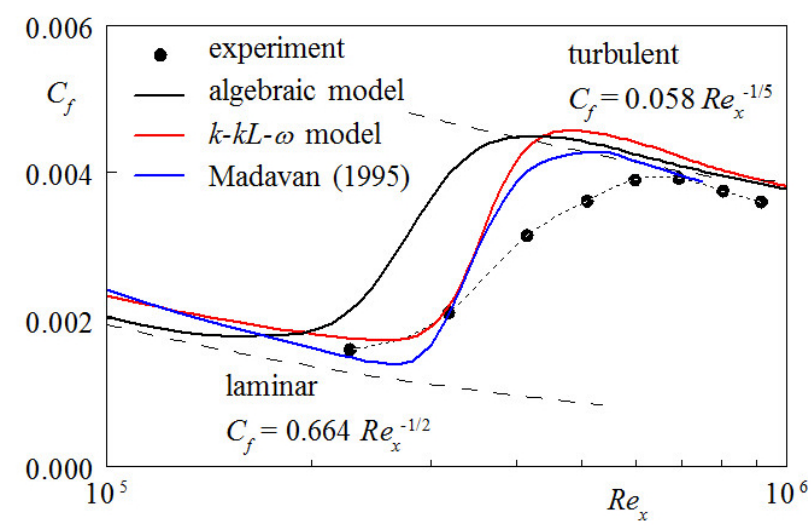

Fig. 1 Skin friction distribution on the heated flat plate

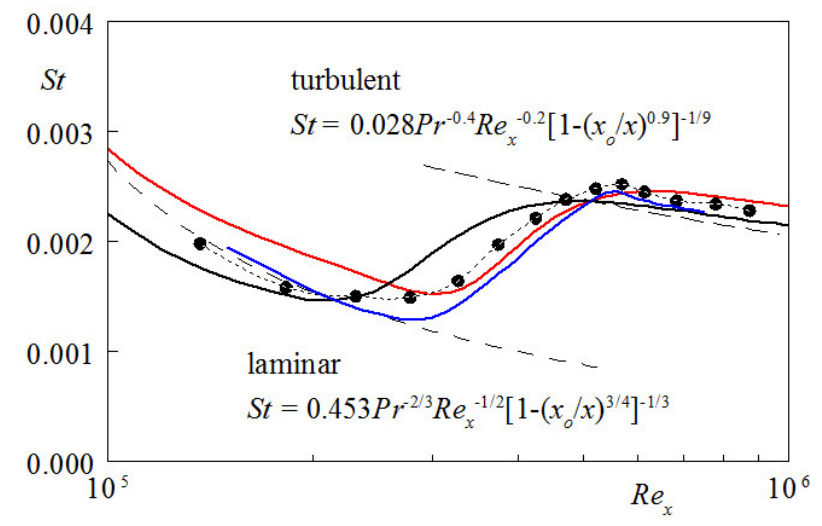

Fig. 2 Distribution of the Stanton number on the heated flat plate

The skin friction distribution on the heated flat plate is compared in Fig. 1 with experimental data of Sohn and Reshotko [16] and with results of numerical simulation by means of the DNS method completed by Madavan [17]. For comparison, well-known skin friction laws for the flat-plate laminar and turbulent boundary layers are shown as well.

It is surprising that the algebraic transition model gives the skin friction distribution with a rather early onset of the transition. The $k-k L-\omega$ model and the DNS method agree well with experimental data, but the predicted length of the transition region is rather shorter.

The heat transfer at the wall is described by the Stanton number determined by the relation

$$
S t=\frac{q_{w}}{\rho c_{p} U_{e}\left(T_{w}-T_{\infty}\right)}
$$

On the basis of the Reynolds analogy, the Stanton number for the constant wall heat transfer $q_{w}=$ const. is given according to Kays and Crawford [5] for the laminar flat-plate boundary layer by the relation

$$
S t_{L}=0.453 \operatorname{Pr}^{-0.67} \operatorname{Re}_{x}^{-0.5}\left[1-\left(\frac{x_{o}}{x}\right)^{0.75}\right]^{-0.333}
$$

and for the turbulent flat-plate boundary layer

$$
S t_{T}=0.028 \operatorname{Pr}^{-0.4} \operatorname{Re}_{x}^{-0.2}\left[1-\left(\frac{x_{o}}{x}\right)^{0.9}\right]^{-0.111}
$$

where $x_{o}$ is the entrance unheated length and the Prandtl number $\operatorname{Pr}=0.708$. The predicted distribution of the Stanton number on the heated flat plate is compared with experimental data of Sohn and Reshotko [16] in Fig. 2. The transition onset predicted by the algebraic model by means of the Stanton number distribution is again slightly upstream similarly as for the skin friction distribution. The $k-k L-\omega$ model gives a good agreement with experimental data comparable with DNS results.

The both models were further applied for the numerical simulation of the compressible flow through the plane VKI high pressure turbine blade cascade with the relative spacing $t / c=0.85$ and the zero inlet flow angle according to measurements of Arts et al. [5]. Experiments were carried out in the test facility enabling an independent selection of both Mach and Reynolds numbers. Besides measurements of the blade velocity distribution, the loss coefficient and exit flow angle, experiments were focused on measurements of the blade convective heat transfer.

Predictions were carried out for two subsonic (MUR 132 and MUR 218) and two transonic regimes (MUR 210 and MUR 213) at the isentropic outlet Reynolds number $R e_{2 i s}=1 \times 10^{6}$, free-stream turbulence $T u=1$ and $4 \%$, and the wall temperature $T_{w}=300 \mathrm{~K}$. Other boundary conditions were prescribed according to individual test cases, i.e. total inlet pressure and total inlet temperature, and the outlet static pressure. The overview of free-stream conditions for selected VKI test cases is given in Table 1.

Table 1 Free-stream conditions for VKI test cases

$\begin{array}{lllll}\text { Case } & \operatorname{Re}_{2 i s} & M_{2 i s} & T u & \mu_{t} / \mu \\ \text { MUR132 } & 0.97 \times 10^{6} & 0.68 & 0.8 & 10 \\ \text { MUR210 } & 1.10 \times 10^{6} & 1.076 & 1 & 10 \\ \text { MUR218 } & 1.01 \times 10^{6} & 0.76 & 4 & 50 \\ \text { MUR213 } & 1.09 \times 10^{6} & 1.068 & 4 & 50\end{array}$

In the case of the $k-k L-\omega$ model, the computational grid containing about 62000 cells is formed by rectangular cells in the boundary layer and triangles and rectangles in the free stream. The nearest node was in the distance from the wall $y^{+} \approx 0.2$. The onset of the grid is in the distance $0.9 c$ upstream of the leading edge. The detail of the computational grid near the trailing edge is shown in Fig. 3.

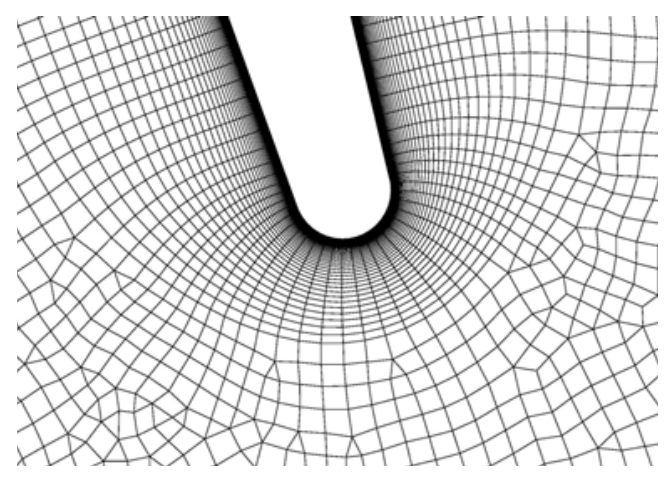

Fig. 3 Detail of the grid near the trailing edge 


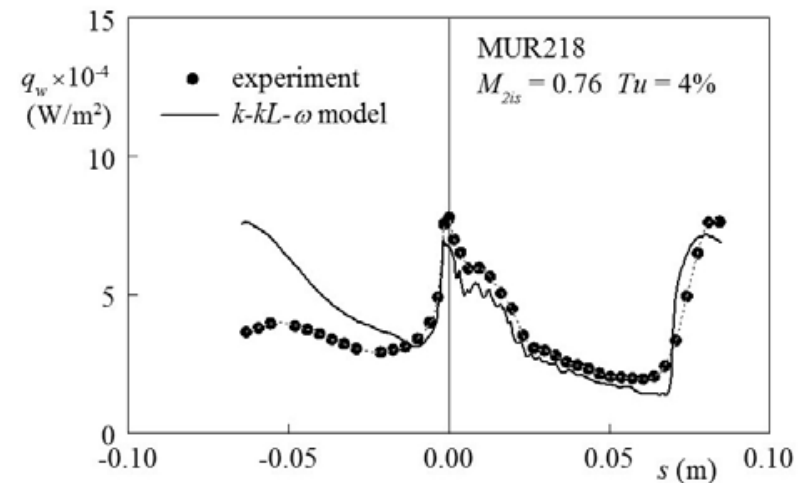

a) MUR 218

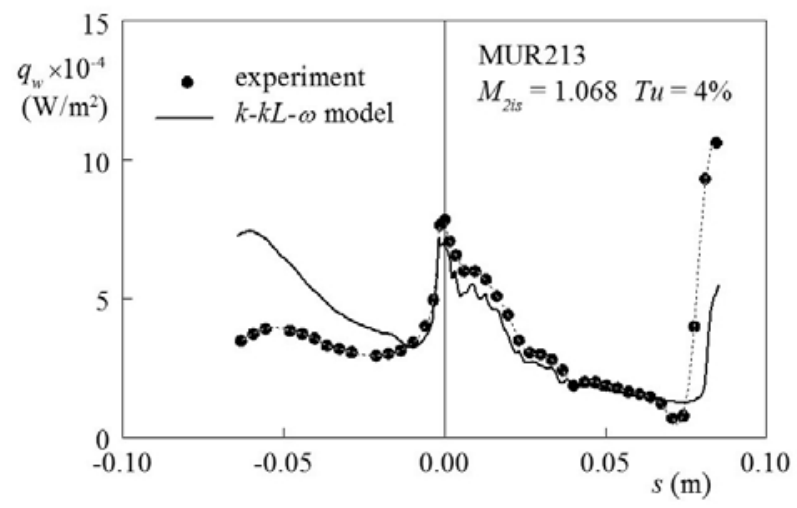

b) MUR 213

Fig. 4 Distribution of the heat flux ( $k-k L-\omega$ model $)$

The distribution of the wall heat flux along the blade obtained by the $k-k L-\omega$ model for the free-stream turbulence about $T u=4 \%$ is shown in Fig. 4. Numerical results are very similar for subsonic and transonic regimes. The agreement with experimental data is quite good on the suction side $(s>0)$ where the transition onset is near upstream of the trailing edge. However, the $k-k L$ $\omega$ model overestimates the heat flux on the pressure side at higher free-stream turbulence.

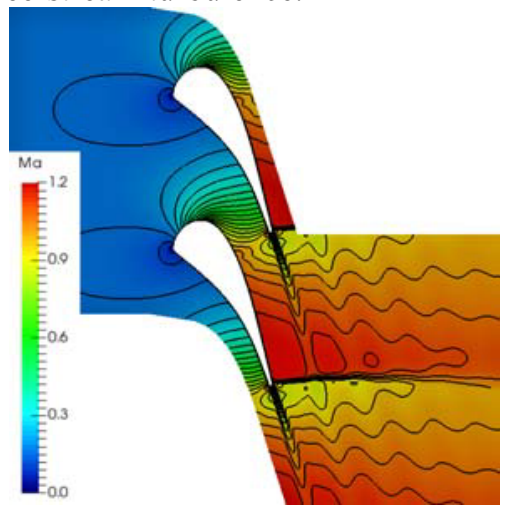

Fig. 5 Isolines of the Mach number (MUR 213)

The Mach number isolines for the case MUR 213 obtained by the $k-k L-\omega$ model are shown in Fig. 5 where the shock wave is apparent at the suction side near upstream of the trailing edge.

The distribution of the wall heat flux obtained by the $k-k L-\omega$ model for the free-stream turbulence about $T u \approx$ $1 \%$ is shown in Fig. 6. For the lower level of free-stream turbulence, the agreement of numerical results with experiment is quite good on the pressure and suction side, but the transition onset on the suction side in the case
MUR 132 is predicted as early as at $s \approx 0.06 \mathrm{~m}$. The difference between the prediction and experimental data can be caused by the bypass-transition threshold function which is not dependent on the pressure gradient.

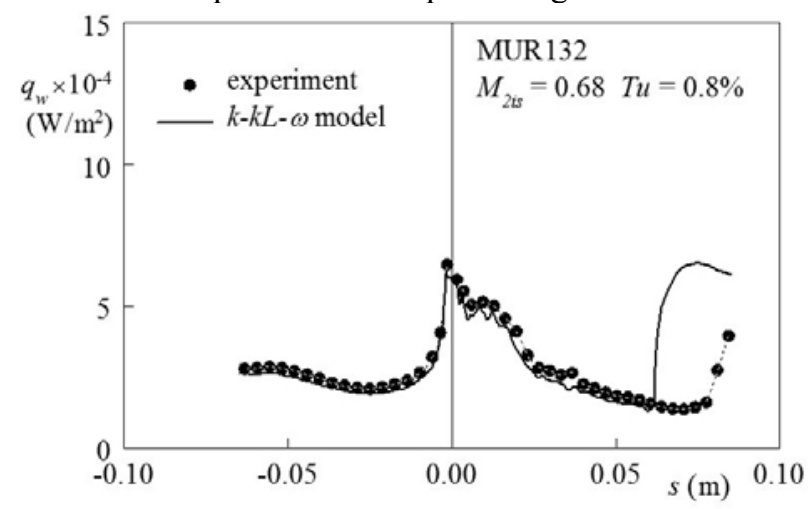

a) MUR 132

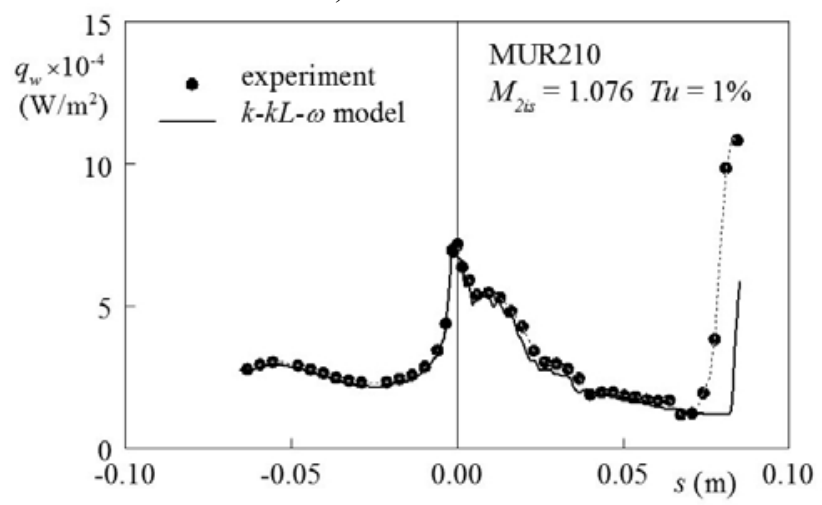

b) MUR 210

Fig. 6 Distribution of the heat flux ( $k-k L-\omega$ model)

Numerical simulations of compressible flow through the VKI turbine blade cascade by the EARSM model completed by the algebraic transition model were carried out using the computational structured mesh with a block overlapping containing about 55000 quadrilateral cells consists of the O-type block around the blade profile and the H-type block which covers one pitch of blade cascade. The detail of the grid near the leading edge is shown in Fig. 7.

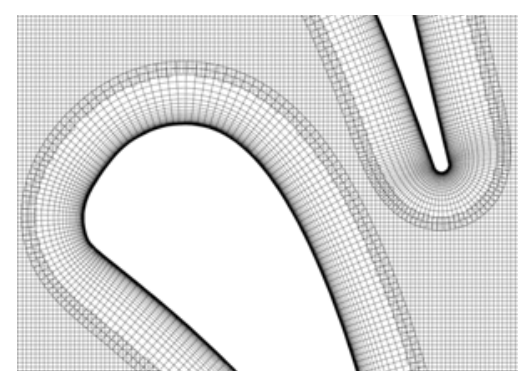

Fig. 7 Detail of the multi-block structured grid

Due to finite non-negligible thickness of the blade trailing edge, simulations were carried out both by the RANS and by the URANS approaches. Predictions of the compressible flow with heat transfer were accomplished for test cases given in Tab. 1 .

The distribution of the wall heat flux obtained by the EARSM model with the algebraic transition model for the free-stream turbulence $T u=4 \%$ and $1 \%$ is shown in 
Fig. 8 and 9. The distribution of the wall heat flux is given for the averaged URANS solution. A very good agreement with experimental data was obtained on the pressure and suction sides for both subsonic regimes where the RANS and URANS approaches give the practically same results.

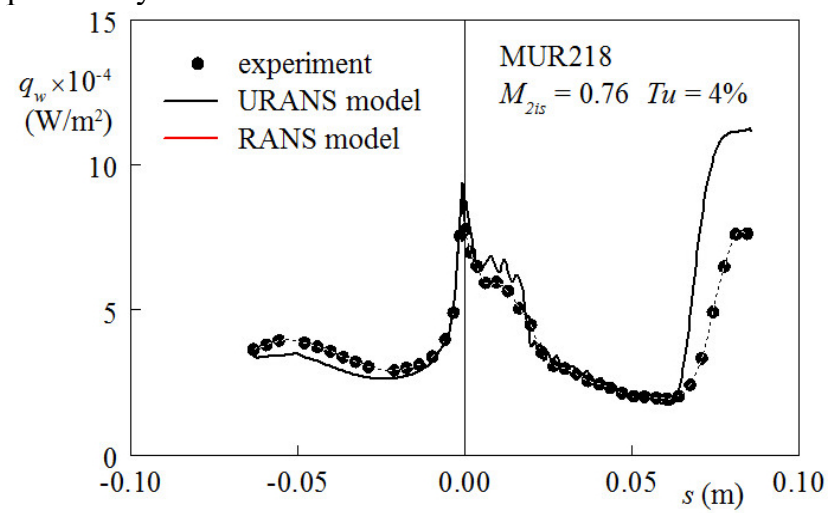

a) MUR 218

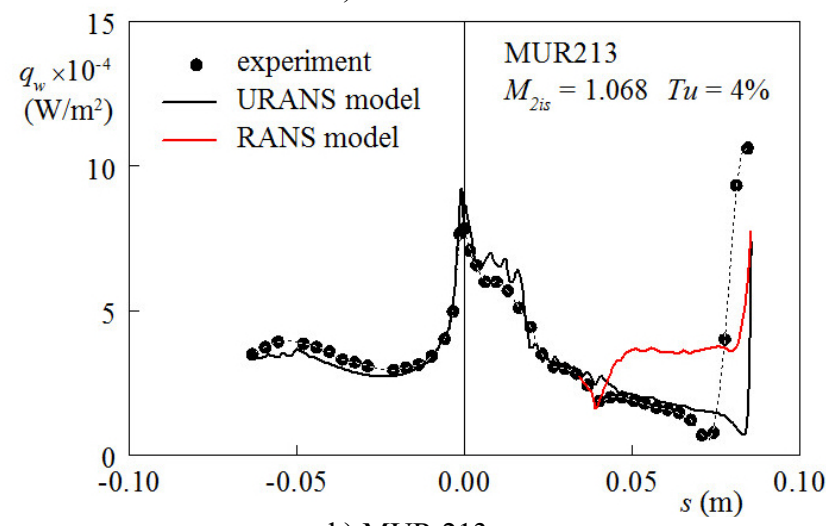

b) MUR 213

Fig. 8 Distribution of the heat flux (algebraic model)

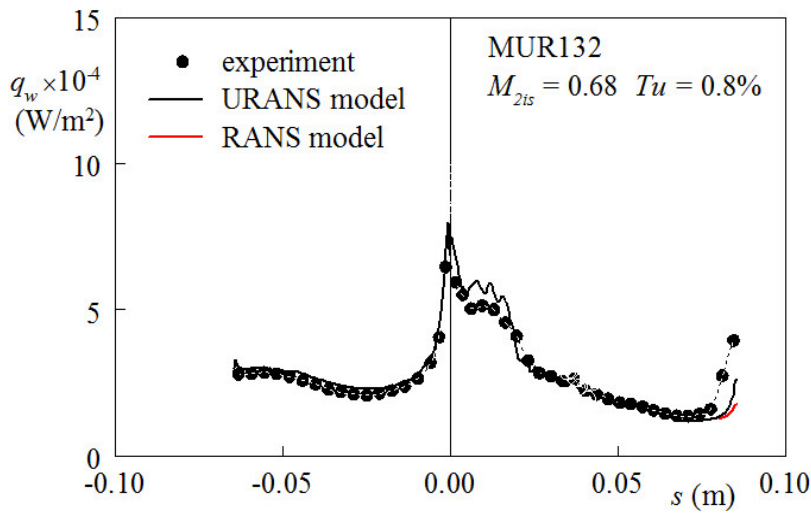

a) MUR 132

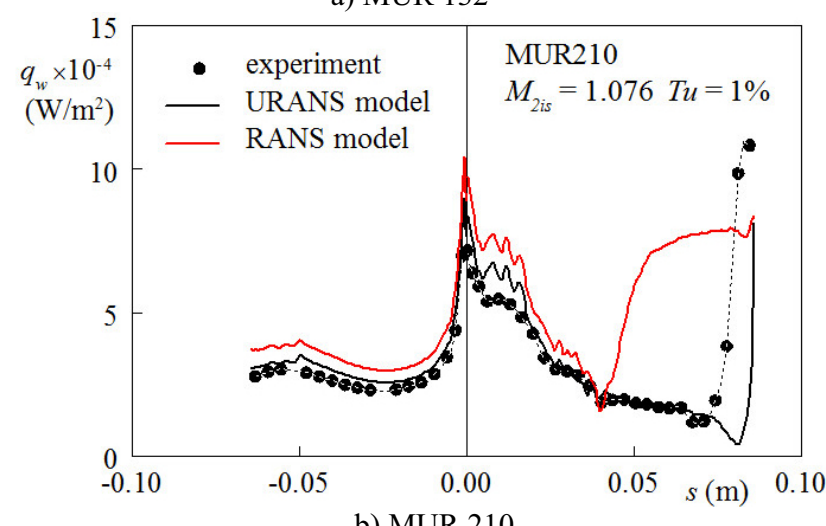

Fig. 9 Distribution of the heat flux (algebraic model)
The flow field in the blade cascade is demonstrated by isolines of the Mach number shown in Fig.10 for the RANS solution (a), the time-averaged URANS solution (b) and the instantaneous URANS solution (c).

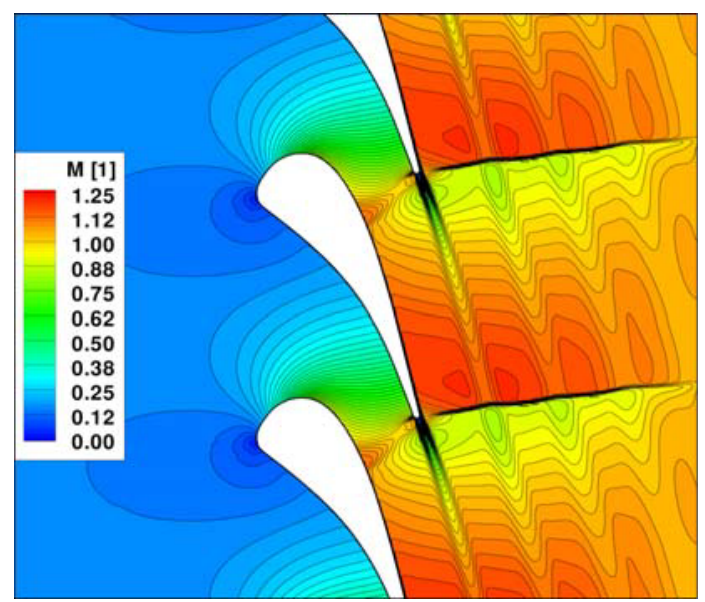

a) RANS solution

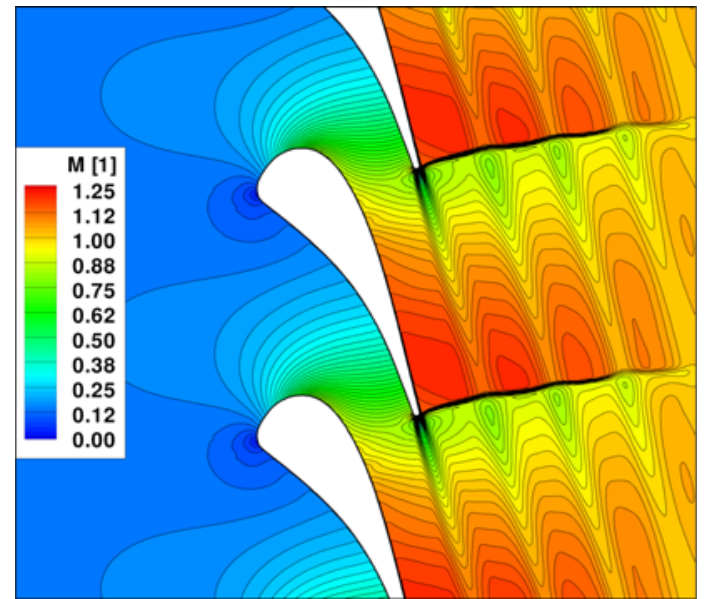

b) URANS time-averaged solution

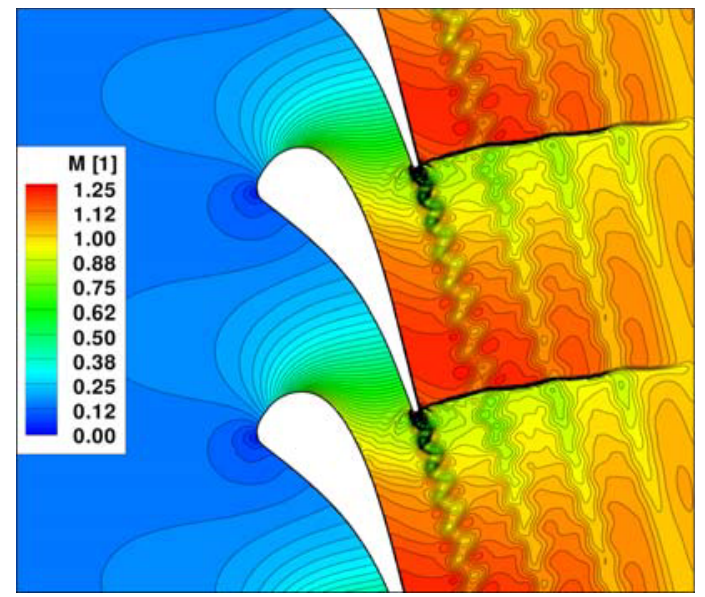

c) URANS instantaneous solution

Fig. 10 Isolines of the Mach number (MUR 213)

The RANS approach gives the transition onset on the suction side at the distance about $s \approx 0.04 \mathrm{~m}$ for the both transonic regimes MUR 213 and MUR 210 similarly as the $\gamma$-Re model of Langtry and Menter [6] for another VKI test case. This transition onset can be caused by the interaction of the boundary layer with the inner branch of the exit shock wave visible for the RANS solution (see 
Fig. 10a) while the time-averaged URANS solution does not show this shock wave.

The URANS approach gives a better agreement with experimental data even though the transition onset on the suction side at $s \approx 0.083 \mathrm{~m}$ is probably caused by the interaction with the exit shock wave. Besides, the instantaneous field of the Mach number isolines obtained by the URANS method shows the noticeable vortex line behind the trailing edge of the blade (see Fig. 10c). Similar flow structures including vortex shedding were found out by the RANS and LES simulations of flow with heat transfer in the VKI blade cascade accomplished by Gourdain et al. [18].

\section{Conclusions}

The transitional flow with heat transfer was simulated by the $k-k L-\omega$ model of Walters and Cokljat [3] and by the EARSM model of Hellsten [1] completed by the algebraic transition model of Straka and Př́ihoda [2] and the turbulent heat transfer model based on the generalized gradient hypothesis. The both mathematical models were tested by experimental data of Sohn and Reshotko [16] for the boundary-layer flow on a heated plat plate and then applied to the simulation of compressible flow through the VKI turbine blade cascade according to measurements of Arts et al. [4].

The simulations were carried out for subsonic and transonic regimes at various values of free-stream turbulence. Due to finite thickness of the blade trailing edge, the RANS and URANS approach was applied for the EARSM model with the algebraic transition model. A good agreement was obtained by both approaches for subsonic regimes but only the URANS approach gives adequate results of numerical simulations for transonic regimes where the RANS approach gives a pseudo shock wave whose interaction with the boundary layer leads to the premature transition on the suction side.

\section{Acknowledgement}

The work was supported by the institutional support RVO 61388998 and by grants TA CR TA03020277 and GA CR P101/12/1271.

\section{References}

1. A. Hellsten, New two-equation turbulence model for aerodynamics applications, PhD. Thesis, Helsinki University of Technology (2004)

2. P. Straka, J. Příhoda, Proc. Conf. Experimental Fluid Mechanics, Liberec, 636-641 (2010)

3. D. K. Walters, D. Cokljat, Jour. Fluids Engineering., 130, 121401-121414 (2008)

4. T. Arts, M. Lambert de Rouvroit, A. W. Rutheford, Aero-thermal investigation of a highly loaded transonic linear turbine guide vane cascade, Technical Note 174, von Karman Institute for Fluid Dynamics, Rhode Saint Genèse (1990)
5. H. Thermann, R. Niehuis, Jour. Turbomachinery, 128, 474-483 (2006)

6. R. Langtry, F. R. Menter, AIAA Jour., 47, 28942906 (2009)

7. S. Kubacki, K. Lodefier, R. Zarzycki, W. Elsner, E. Dick, Flow, Turbulence and Combustion, 83, 539568 (2009)

8. F. R. Menter, P. E. Smirnov, T. Liu, R. Avancha, Flow, Turbulence Combustion, 95, 583-619 (2015)

9. B. E. Launder, Jour. Heat Transfer, 110, 1112-1128, (1988)

10. W. M. Kays, M. B. Crawford, Convective heat and mass transfer, McGraw Hill, New York (1993)

11. F. R. Menter, AIAA Jour., 32, 1598-1605 (1994)

12. R. Narasimha, Prog. Aerospace Science, 22, 29-80, (1985)

13. W. J. Solomon, G. J. Walker, J. P. Gostelow, Jour. Turbomachinery, 118, 744-751 (1996)

14. R. E. Mayle, Jour. Turbomachinery, 113, 509-537 (1993)

15. J. Fürst, J. Př́ihoda, P. Straka, Computing, 95, S163S182 (2013)

16. K.-H. Sohn, E. Reshotko, Experimental study of boundary layer transition with elevated free-stream turbulence on a heated fiat plate, NASA CR-187068 (1991)

17. N. K. Madavan, Direct numerical simulation of transitional and turbulent flow over a heated flat plate using finite-difference schemes, NASA CR-200759 (1995)

18. N. Gourdain, L. Y. M. Gicquel, E. Collado Morata, Jour. Propulsion and Power, 2, 847-862 (2011) 